\title{
High dilutions of Magonia pubescens hidrogel affect germination variables in Sorghum bicolor L. Moench
}

\author{
Carlos Moacir Bonato, Beatriz Rigon, Aleandro Ferreira de Souza, \\ Carolina Bertuzzi Pereira, Bruno Reis.
}

State University of Maringá, Maringá, PR, Brazil

\begin{abstract}
Introduction: In science homeopathic diseases or physiological disorders are not considered just a result of abiotic and biotic factors, but rather a consequence of loss of organic system homeostasis. Homeopathic science is currently being used efficiently in the control of plagues [1], plant diseases[2], in the increase of medicinal plants' active principles[3] and in plant metabolism[4,5]. Although actual results, both in the academic and field-level, very little is known about physiological mechanisms action of homeopathic medicine on germination process[6]. This work aims to study the effect of $M$. pubescens hydrogel, on some physiological variables of sorghum germination (Sorghum bicolor L. Moench).
\end{abstract}

Material and methods: The experiment was conducted at Homeopathy and Plant Physiology of Biology Department at UEM in the period from 04/05/06 to 30/12/06. M. pubescens (tingui) seeds were obtained from the region of Montes Claros - Minas Gerais. The M. pubescens hidrogel was obtained from the external centrals wrappers of 4 dry seeds, after they have been disposed in petri dishes with distilled water for a period of 36 hours of soaking $\left(25^{\circ} \mathrm{C}\right)$. The hydrogel mother tincture was prepared according to Manual of Technical Standards for Homeopathic Drugstore[7] 3rd ed (2003), in the proportion of a hidrogel part (5g) to ten parts $(50 \mathrm{~g})$ of absolute alcohol $70 \%$ and stored in a glass amber (capped and protected from light). After 15 days of maceration, the solution was filtered and after $48 \mathrm{~h}$ at rest, the mother tincture was considered ready for use. The dilution $1 \mathrm{cH}$ (Centesimal Hahnemannian) was obtained by adding $0.2 \mathrm{ml}$ of the mother tincture in 19.8 $\mathrm{ml}$ of distilled water (1/100) and sucussioned 100 times (33 sucussions $\mathrm{s}^{-1}$ ) by mechanical arm dynamizer with automatic stop (Model Denise 50 - AUTIC). The subsequent dilutions (2cH to $30 \mathrm{cH}$ ) were obtained from the same procedure, starting from the dilution $1 \mathrm{cH}$.

Bioassay: In petri dish containing 15 seeds of sorghum in a circular distributed were added $10 \mathrm{ml}$ with their dilutions $(3,6,9,12,15,20$ and $30 \mathrm{cH})$ and the control containing distilled water. The petri dishes were placed in a growth chamber (type BOD), temperature of $(25 \pm 2)^{\circ} \mathrm{C}$ and photoperiod of $16 \mathrm{~h}$. The variables were analyzed by germination period of $73.5 \mathrm{~h}$ as described below:

- Germination $(\% \mathrm{G}): \% G=\left(\sum n_{i} \cdot N^{-1}\right) \times 100$, where $\sum n_{i}$, is the total number of germinated seeds in relation to the number of seeds put to germinate, expressed in percentage;

- Germination average time (GAT): $G A T=\sum n_{i} . t_{i} / \sum n_{i}$, where $n_{i}$ is the number of germinated seeds within a certain interval of time $t_{i-1}$ and $t_{i}$; expressed in hours.

- Germination average speed (GAS), expressed in hours): GAS $=\sum n_{i} / \sum n_{i} . t_{i}$ 
- Germination speed index (GSI): $I V G=G_{1} / N_{1}+G_{2} / N_{2}+$ $G_{n} / N n$, where $\mathrm{G}_{1}, \mathrm{G}_{2} \ldots G_{n}$ is the number of germinated seeds and $\mathrm{N}_{1}, \mathrm{~N}_{2}, \ldots \mathrm{N}_{\mathrm{n}}$ is the number of hours after sowing.

The total number of germinated seeds, at each time (12h) was also analyzed. Seeds were considered germinated when the radicle had 1 to $2 \mathrm{~mm}$ of lengths.

Experimental design: The experimental design was randomized block with 4 replications, totaling 32 experimental units. It was adopted the double-blind methodology, to avoid possible interference or direction by the researcher.

Statistical Analysis: The data were analyzed by ANOVA and the averages compared by Scott-Knott test $(\mathrm{p} \leq 0.05)$. The twinning combined data were analyzed for interaction germination $\mathrm{x}$ time $(\mathrm{G} \times \mathrm{T})$ by $\mathrm{F}$ test to $5 \%$ of probability.

Results and discussion: The homeopathy of Magonia pubescens hydrogel affected on the germination kinetic variables of sorghum seeds, when compared with the control (Fig. 1). This effect was most observed in the initial process of germination (from 13h).

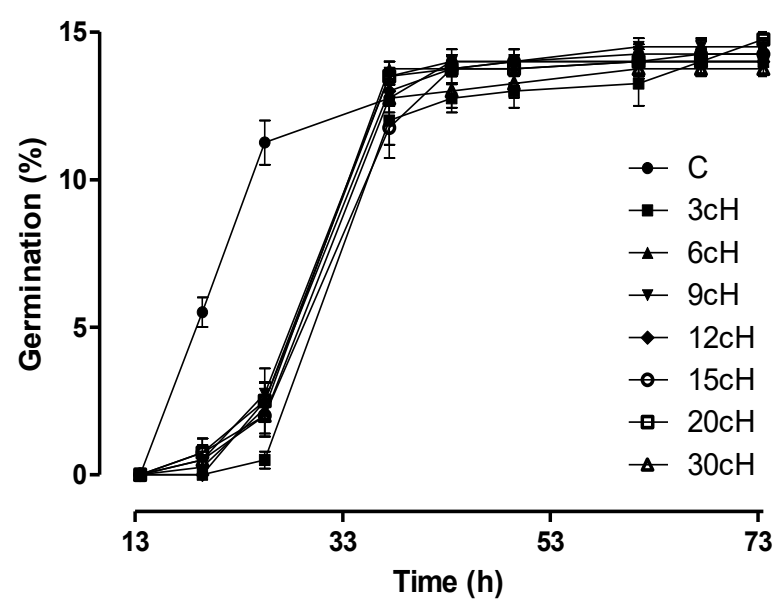

Figure 1 - Percentage of sorghum germinated seeds in function of time. $(\mathrm{P}<0.001$ by $\mathrm{F}$ test). $\mathrm{C}=$ control.

Research accomplished by Salgado-Labouriau (1973) [8] showed that the hydrogel formed from the external wrapper Magonia pubescens seeds, does not contain inhibiting, but contains factors that accelerate the germination process. Apparently, these results seem contradictory. However, for the homoeopathic optics, some used medicines in a considered way might have determined effect. Already in high diluted doses this behavior can be reversed, as it happens with some drugs. This behavior in pharmacokinetics is denomined Hormesis. When diluted and given dynamism, the product of hydrogel, instead of stimulating, it can delay the germination for the same phenomenon. Hormesis is not yet explained by science. Homeopathy of the Magonia pubescens gel significantly increased the germination average time (GAT) of sorghum seeds and reduced the germination average speed (GAS) and the germination speed index (GSI) (Fig. 2A, B and C). The values of these variables suggest that homeopathy, somehow slowed the speed of sorghum seeds soaking. 

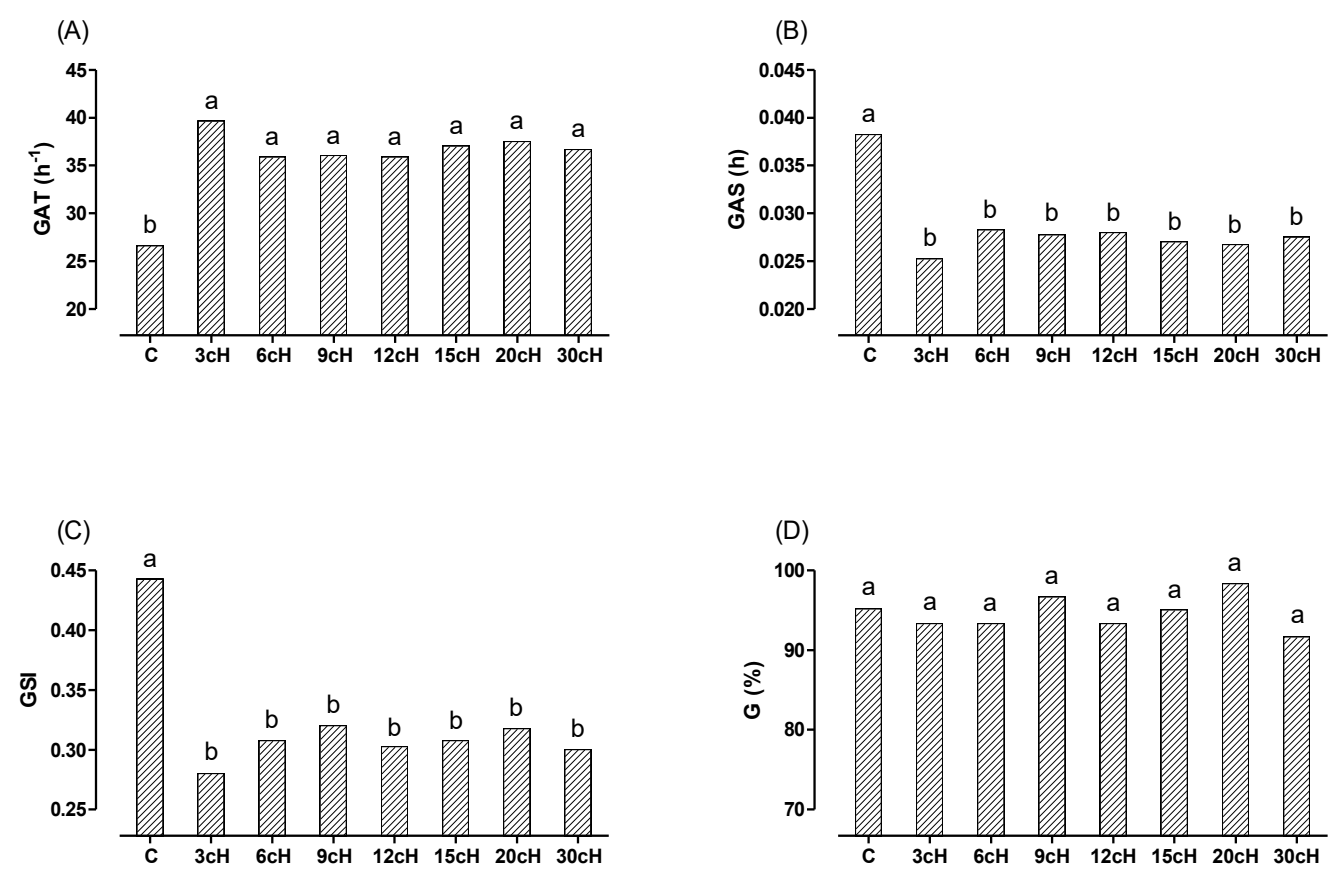

Figure 2 - Germination average time (A), germination average speed (B), germination average index (C) and germination percentage (D) of sorghum seeds. $(\mathrm{P} \leq 0.001)$ by Scott Knott test). $\mathrm{C}=$ control.

Conclusion: The results here presented suggest that high dilutions of Magonia pubescens hidrogel can be used in future experiment such as bioherbicide.

Keywords: Tingui, seedlings, homeopathy, double-blind.

\section{References}

[1] Cavalca PAM, Lolis MIGA, Reis B, Bonato CM. Homeopathic and Larvicide Effect of Eucalyptus cinerea Essential Oil against Aedes aegypti. Brazilian Archives of Biology and Technology. 2010; 53(4): 835-843.

[2] Shah-Rossi D, Heusser P, Baumgartner S. Homeopathic treatment of Arabidopsis thaliana plants infected with Pseudomonas syringae. The Scientific World Journal.TSW Holistic Health \& Medicine. 2009; 9: 320-330. DOI $10.1100 /$ tsw.

[3] Bonato CM, Proença GT, Reis B. Homeopathic drugs Arsenicum album and Sulphur affect the growth and essential oil content in mint (Mentha arvensis L.). Acta Scientiarum Agronomy. 2009; 31(1): 101-105.

[4] Betti L, Brizzi M, Nani D, Peruzzi M. Effect of high dilutions of Arsenicum album on wheat seedlings from seeds poisoned with the same substance. British Homeopathic Journal. 1997; 86(2): 86-89.

[5] Bonato CM, Silva EP. Effect of the homeopathic solution Sulphur on the growth and productivity of radish. Acta scientiarum. 2003; 25: 259-263.

[6] Bonato CM. Homeopathy in the host physiology. Fitopatologia Brasileira. 2007; 32: 79-82. 
[7] Manual de Normas Técnicas para Farmácia Homeopática: ampliação dos aspectos técnicos e práticos para farmácia homeopática. 2003. 3ed. Curitiba. [portuguese]

[8] Salgado-Labouriau ML. A semente de Magonia pubescens St. Hil. - Morfologia e germinação. Anais da Academia Brasileira de Ciências. Rio de Janeiro. 1973; 45(3/4): 501-537. [portuguese]

\title{
Altas diluições de hidrogel de Magonia pubescens afetam as variáveis de germinação de Sorghum bicolor L. Moench
}

\begin{abstract}
RESUMO
Introdução: $\mathrm{Na}$ ciência homeopática as doenças ou distúrbios fisiológicos não são considerados apenas resultado de fatores abióticos e bióticos, mas sim uma consequência da perda de homeostase no sistema orgânico. Atualmente a ciência homeopática está sendo usada de forma eficiente no controle de pragas [1], doenças de plantas [2], no aumento de princípios ativos de plantas medicinais [3] e no metabolismo das plantas [4,5]. Apesar dos resultados obtidos atualmente, tanto no âmbito acadêmico quanto em nível de campo, muito pouco se sabe sobre a ação fisiológica dos medicamentos homeopáticos no processo de germinação [6] entre outros. Este trabalho visa estudar o efeito do hydrogel de $M$. pubescens, em algumas variáveis fisiológicas de germinação de sorgo (Sorghum bicolor L. Moench).

Material e métodos: O experimento foi conduzido no laboratório de Homeopatia e Fisiologia Vegetal do Departamento de Biologia da UEM no período de 04/05/06 a 30/12/06. As sementes de M. pubescens (tingui) foram obtidas da região de Montes Claros - Minas Gerais. O hidrogel de M. pubescens foi obtido dos envoltórios externos centrais de 4 sementes secas, que foram dispostos em placas de Petri com água destilada por um período de 36 horas de imersão $\left(25^{\circ} \mathrm{C}\right)$. A tintura-mãe de hidrogel foi preparada de acordo com o Manual de Normas Técnicas para Farmácia Homeopática [7] $3^{a}$ ed (2003), na proporção de uma parte de hidrogel (5g) para dez partes (50g) de álcool absoluto $70 \%$ e armazenados em frasco âmbar (tampado e protegido da luz). Após 15 dias de maceração, a solução foi filtrada e após $48 \mathrm{~h}$ em repouso, a tintura mãe foi considerada pronta para uso. A diluição $1 \mathrm{cH}$ (Centesimal Hahnemanniana) foi obtida pela adição de $0,2 \mathrm{ml}$ da tintura-mãe em 19,8 ml de água destilada (1/100) e sucussionada 100 vezes (33 sucussões $\mathrm{s}^{-1}$ ) com dinamizador braço mecânico com parada automática (Modelo Denise 50 - AUTIC). As diluições subsequentes $(2 \mathrm{cH}$ a $30 \mathrm{cH})$ foram obtidos a partir do mesmo procedimento, a partir da $1 \mathrm{cH}$.
\end{abstract}

Bioensaio: Em placa de Petri contendo 15 sementes de sorgo foram distribuídas de forma circular e adicionados $10 \mathrm{ml}$ das diluições (3, 6, 9, 12, 15, 20 e 30cH). O controle foi constituído de água destilada. As placas de Petri foram colocadas em câmara de crescimento (tipo BOD), a temperatura de $(25 \pm 2)^{\circ} \mathrm{C} \mathrm{e}$ fotoperíodo de $16 \mathrm{~h}$. As variáveis foram analisadas por período de germinação de $73,5 \mathrm{~h}$ conforme descrito abaixo: 
- A germinação (\%G): \%G $=\left(\sum n_{i} \cdot N^{-1}\right)$ x 100 , em que: $\sum n_{i}$, é o número total de sementes germinadas em relação ao número de sementes colocadas para germinar, expressa em percentagem;

- Tempo médio de germinação (TMG): $T M G=\sum n_{i} . t_{i} / \sum n_{i}$, em que $n_{i}$ é o número de sementes germinadas dentro de um certo intervalo de tempo $t_{i-1}$ e $t_{i}$; expresso em horas.

- A velocidade média de germinação (VMG), expresso em horas: $\mathrm{VMG}=\sum n_{i} / \sum n_{i} . t_{i}$

- Índice de velocidade de germinação (IVG): $I V G=G_{1} / N_{l}+G_{2} / N_{2}+\ldots \ldots . . G_{n} / N n$, onde $\mathrm{G}_{1}$, $\mathrm{G}_{2} \ldots G_{n}$ é o número de sementes germinadas e $\mathrm{N} 1, \mathrm{~N} 2, \ldots \mathrm{Nn}$ é o número de horas após a semeadura.

O número total de sementes germinadas, em cada momento (12h) também foi analisada. As sementes foram consideradas germinadas quando a radícula tinha 1-2 $\mathrm{mm}$ de comprimento.

Delineamento experimental: O delineamento experimental foi em blocos casualizados com quatro repetições, totalizando 32 unidades experimentais. Foi adotada a metodologia de duplo-cego, para evitar possíveis interferências dos pesquisadores.

Análise estatística: Os dados foram analisados por ANOVA e as médias comparadas pelo teste de ScottKnott $(\mathrm{p} \leq 0,05)$. Os dados combinados de geminação foram analisados para a interação germinação $\mathrm{x}$ tempo (G x T) pelo teste $\mathrm{F}$ a $5 \%$ de probabilidade.

Resultados e discussão: A homeopatia do hidrogel de Magonia pubescens afetou a cinética das variáveis de germinação de sementes de sorgo, quando comparado com o controle (Fig. 1). Este efeito foi mais acentuado no processo inicial de germinação (das 13h).

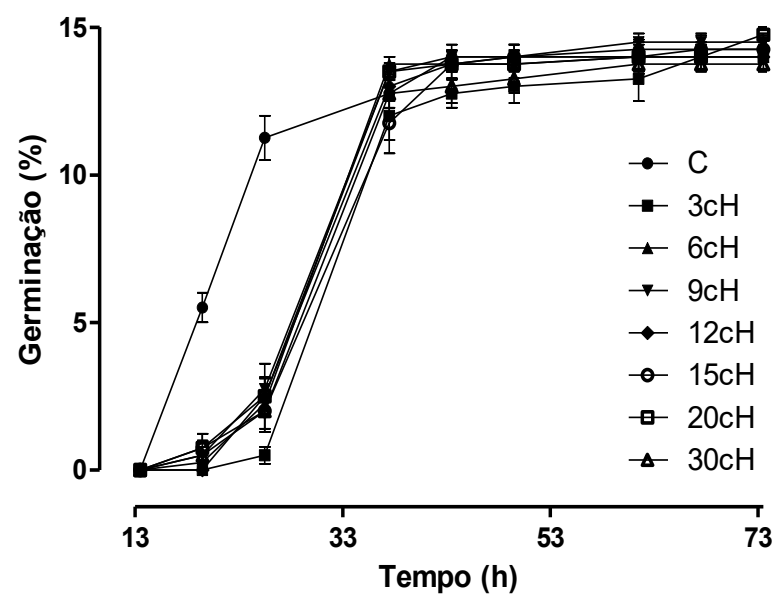

Figura 1 - Porcentagem de germinação de sementes de sorgo em função do tempo. ( $\mathrm{P}<0.001$ by $\mathrm{F}$ test). $\mathrm{C}=$ controle.

Pesquisa realizada por Salgado-Labouriau (1973) [8] mostraram que o hidrogel formado a partir do envoltório exterior de sementes de Magonia pubescens não contém inibidor, mas contém fatores que aceleram o processo de germinação. Aparentemente, esses resultados parecem contraditórios. No entanto, na visão homeopática, alguns medicamentos utilizados desta forma podem apresentar este efeito. Já em altas diluições esse 
comportamento pode ser revertido, como acontece com alguns medicamentos. Esse comportamento em farmacocinética é denominado efeito hormese. O efeito hormese ainda não foi explicado pela ciência.

Homeopatia do hidrogel da Magonia pubescens aumentou significativamente o tempo médio de germinação (TMG) de sementes de sorgo e reduziu a velocidade média de germinação (VMG) e o índice de velocidade de germinação (IVG) (Fig. 2A, B e C). Os valores dessas variáveis sugerem que a homeopatia, de alguma forma, diminuiu a velocidade germinação de sementes de sorgo.
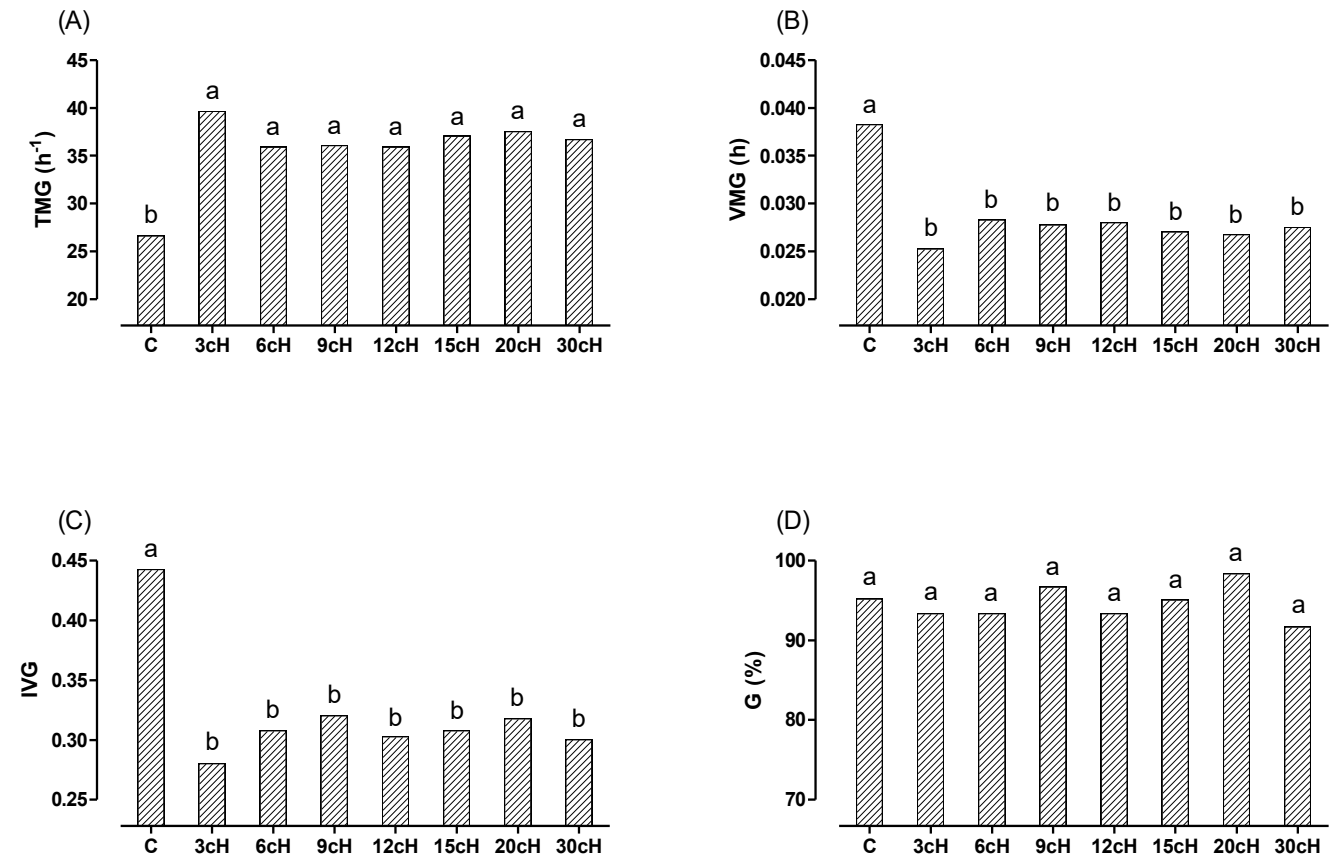

Figura 2 - Tempo médio de germinação (A), velocidade média de germinação (B), índice de velocidade de germinação $(\mathrm{C})$ e porcentagem de germinação $(\mathrm{D})$ de sementes de sorgo. $(\mathrm{P} \leq 0.001)$ pelo teste Scott Knott). $\mathrm{C}=$ controle.

Conclusão: Os resultados apresentados sugerem que altas diluições de hidrogel de Magonia pubescens podem ser usadas em futuros experimentos como bioherbicida.

Palavra-chave: Tingui, germinação, homeopatia, duplo-cego.

\section{(c) BY-NC-ND Licensed to GIRI}

Support: authors declare that this study received no funding

Conflict of interest: authors declare there is no conflict of interest

Correspondence author: Carlos Moacir Bonato, cmbonato@uem.br or cmbonato@hotmail.com

How to cite this article: Bonato CM, Rigon B, de Souza AF, Pereira CB, Reis B. High dilutions of Magonia pubescens hidrogel affect germination variables in Sorghum bicolor L. Moench. Int J High Dilution Res [online]. 2011 [cited YYYY Month dd]; 10(36): 253-258. Proceedings of the XXV GIRI Symposium and VIII CBFH; 2011 Sep 04-07; Foz do Iguaçu (Brazil). GIRI and ABFH; 2011; Available from: http://www.feg.unesp.br/ ojs/index.php/ijhdr/article/view/512/526 